\title{
On Aggregation Operators of Transitive Similarity and Dissimilarity Relations
}

\author{
Jorge Orozco \\ Software Department \\ Technical University of Catalonia \\ Barcelona, Spain \\ E-mail: jorozco@1si.upc.es
}

\author{
Lluís Belanche \\ Software Department \\ Technical University of Catalonia \\ Barcelona, Spain \\ E-mail: belanche@1si.upc.es
}

\begin{abstract}
Similarity and dissimilarity are widely used concepts. One of the most studied matters is their combination or aggregation. However, transitivity property is often ignored when aggregating despite being a highly important property, studied by many authors but from different points of view. We collect here the most recent results in preserving transitivity when aggregating, intending to clarify the relationship between aggregation and transitivity and making it useful to design aggregation operators that keep transitivity property. Some examples of the utility of the results are also shown.
\end{abstract}

\section{INTRODUCTION}

Similarity and dissimilarity relations are commonly used in many fields of Artificial Intelligence. Nevertheless, it is a hard concept to establish as illustrated by the several different definitions present in the literature [1], [2], [3], [4]. Many of their properties are under discussion and one of them is transitivity.

Also, one of the fundamental aspects of these relations is aggregation and how it keeps their properties. Many authors have studied the effects of aggregation on transitivity [5], [6], [7], [1], [8], [9], [10]. This important field is studied by many authors but from different points of view. We collect here some of their most relevant results, trying to put them in a format that could be useful to design or choose aggregation operators that keep any transitivity. These results are a generalization of those achieved with metrics, since metrics are a particular case of dissimilarity relations. We present some practical examples that can help to show the importance of this matter.

The paper is structured as follows. In the next section, we introduce briefly a definition for similarity and dissimilarity relations. In section III we introduce the aggregation, its definition, properties and the different types of aggregation operators. Next, in section IV we explain the relationship between aggregation and transitivity and we present the results in this field. In section V some examples are introduced to show the effects of the theorems. Finally, in section VI some conclusions about this issue are expressed.

\section{SIMILARITY AND DISSIMILARITY RELATIONS}

Although they are widely used concepts, there are several definitions of similarity and dissimilarity relations [2], [4], [11]. In general, all authors agree that similarity and dissimilarity relations measure the differences between two objects.
However, their definition, properties and even their application domain differs. Let us define a similarity and a dissimilarity as generalizations of a fuzzy equivalence relation (or unequivalence, in case of dissimilarity). Besides, we consider that a dissimilarity complements the definition of similarity because both are dual concepts. Next we define both similarity and dissimilarity and we introduce their properties.

Let $X$ be a non-empty set where there is defined an equality relation $\stackrel{X}{=}$. Let $s$ be a function

$$
s: X \times X \longrightarrow I \subset \mathbb{R}
$$

Assume that $s$ is upper bounded, exhaustive and total. This implies that $I$ is upper bounded and also that sup $I$ exists ${ }^{1}$.

Analogously, a dissimilarity function is defined as follows: Let $\delta$ be a function

$$
\delta: X \times X \longrightarrow I \subset \mathbb{R}
$$

Also assume that $\delta$ is lower bounded, exhaustive and total. In this case, $I$ is lower bounded and inf $I$ exists.

Define $s_{\text {max }} \equiv \sup _{\mathbb{R}} I$ and define $\delta_{\min } \equiv \inf _{\mathbb{R}} I$. Without loss of generality, consider $s_{\max } \geq 0$ and $\delta_{\min } \geq 0$. In any other case, a non-negative maximum or minimum can be obtained applying some transformation (e.g. $s+\left|s_{\max }\right|$ ).

Functions $s$ and $\delta$ can satisfy the following axioms, for any $x, y, z \in X$ :

- Reflexivity.

$$
\begin{aligned}
& -s(x, x)=s_{\max } . \text { This implies } \sup _{\mathbb{R}} I \in I . \\
& -\delta(x, x)=\delta_{\text {min }} . \text { This implies } \inf _{\mathbb{R}} I \in I .
\end{aligned}
$$

- Strong reflexivity.

$$
\begin{aligned}
& -s(x, y)=s_{\max } \Longleftrightarrow x \stackrel{x}{=} y . \\
& -\delta(x, y)=\delta_{\text {min }} \Longleftrightarrow x \stackrel{x}{=} y .
\end{aligned}
$$

- Symmetry.

$-s(x, y)=s(y, x)$.

- $\delta(x, y)=\delta(y, x)$.

- Transitivity.

${ }^{1}$ In this document we will only focus on similarities and dissimilarities whose images are subsets of $\mathbb{R}$. For a more general view see [12]. 
- For all three elements $x, y, z \in X$, there is a similarity transitivity operator $\tau_{\Sigma}$ such that fulfills the following inequality:

$$
s(x, y) \geq \tau_{\Sigma}(s(x, z), s(z, y))
$$

- For all three elements $x, y, z \in X$, there is a dissimilarity transitivity operator $\tau_{\Delta}$ such that fulfills the following inequality:

$$
\delta(x, y) \leq \tau_{\Delta}(\delta(x, z), \delta(z, y))
$$

Note that there are no definitions of both transitivity operators. Further on, we will see that they are a special type of aggregation operators.

Obviously, there are more properties that can be included in the previous list but they are not relevant for this paper. Usually similarity and dissimilarity properties are source of discussion, specially transitivity property [13], [14], [15]. We hold that transitivity is a fundamental property and a very interesting matter of research.

\section{AGgREGATION OPERATORS}

Although we have started with similarity and dissimilarity relations, the main matter of this paper is aggregation and how it must keep the foregoing properties. Firstly, we must define a similarity or dissimilarity aggregation operator. Again, there is no unique definition and many authors point out different properties [8], [16].

Let us define an aggregation operator as a function $\Theta: I^{n} \longrightarrow I$, where $n \in \mathbb{N}$, and $I$ is a set of similarity or a set of dissimilarity values. Using that both similarity and dissimilarity are functions from $X^{2}$ to $I$, we can consider an aggregation operator as a similarity or a dissimilarity defined from the cartesian product of $n$ sets (i.e. $X_{1}^{2} \times \ldots \times X_{n}^{2}$ ) to $I$. From here on, $\Theta\left[s_{i}\left(x_{i}, y_{i}\right)\right]_{i}$ is the abbreviated notation for $\Theta\left(s_{1}\left(x_{1}, y_{1}\right), \ldots, s_{n}\left(x_{n}, y_{n}\right)\right)$.

Some properties that an aggregation operator can fulfill are the following, where $i \in\{1, \ldots, n\}:^{2}$

- Idempotency. If, $\forall x_{i}, y_{i} \in X_{i}, s_{i}\left(x_{i}, y_{i}\right)=k$, an aggregation operator is idempotent when $\Theta\left[s_{i}\left(x_{i}, y_{i}\right)\right]_{i}=k$.

- Symmetry. $\Theta$ is symmetric if, $\forall x_{i}, y_{i} \in X_{i}$, $\Theta\left[s_{i}\left(x_{i}, y_{i}\right)\right]_{i}=\Theta\left[\sigma\left(s_{i}\left(x_{i}, y_{i}\right)\right)\right]$, for all the permutations $\sigma=\{\sigma(1), \ldots, \sigma(n)\}$ of $\{1, \ldots, n\}$.

- Monotonicity. $\Theta$ is monotonic if $\Theta\left[s_{i}\left(x_{i}, y_{i}\right)\right] \leq$ $\Theta\left[s_{i}^{\prime}\left(x_{i}, y_{i}\right)\right]$, whenever $s_{i}\left(x_{i}, y_{i}\right) \leq s_{i}^{\prime}\left(x_{i}, y_{i}\right)$.

- Associativity. When, for any $p \in\{1, \ldots, n\}$,

$$
\dot{\Theta}\left[s_{i}\left(x_{i}, y_{i}\right)\right]_{i}=\Theta\left(\Theta\left[s_{j}\left(x_{j}, y_{j}\right)\right]_{j}, \Theta\left[s_{k}\left(x_{k}, y_{k}\right)\right]_{k}\right)
$$

with $j \in\{1, \ldots, p\}, k \in\{p+1, \ldots, n\}$.

- Neutral element. $\Theta$ has neutral element if, for a certain value $e$, when $s_{j}\left(x_{j}, y_{j}\right)=e$ (with $j \in\{1, \ldots, n\}$ ) then

$$
\Theta\left(s_{1}, \ldots, s_{n}\right)=\Theta\left(s_{1}, \ldots, s_{j-1}, s_{j+1}, \ldots, s_{n}\right)
$$

${ }^{2}$ We choose here similarity functions to represent aggregation properties. Obviously, dissimilarity case is analogous.
Recall that we did not introduce a definition of transitivity operators. Now, we can define them as a special type of aggregation operators.

Definition 3.1 (Transitive operator): A transitive operator $\tau: I \times I$ is a binary aggregation operator that fulfills symmetry, associativity, monotonicity and neutral element. If $\tau$ is a similarity transitive operator, the monotonicity is nondecreasing and the neutral element is $s_{\max }$. In this case $\tau$ is denoted $\tau_{\Sigma}$. If $\tau$ is a dissimilarity transitivity operator, the monotonicity is non-increasing and the neutral element is $\delta_{\max }$. In this case $\tau$ is denoted $\tau_{\Delta}$.

When $\tau$ is defined in the interval $[0,1]$ it is known as a triangular norm [17]. Thus, any $\tau_{\Sigma}$ defined on [0,1] is a tnorm, and any $\tau_{\Delta}$ defined on $[0,1]$ is a t-conorm. These norms are particular cases of aggregation operators.

If an aggregation of similarities is a similarity and the same for dissimilarities, the original properties of similarities or dissimilarities must be present on the aggregated one. Reflexivity or symmetry are trivial, but transitivity is the most controversial one: For a set of $\tau$-transitive similarities or dissimilarities, not any aggregation operator keeps the $\tau$-transitivity. Actually, what happens then is that the transitivity of the aggregated similarity or dissimilarity is weaker than the transitivity of the original similarities or dissimilarities.

Definition 3.2: Let $T_{\Sigma}$ be the set of all the similarity transitivity operators and $T_{\Delta}$ be the set of all dissimilarity transitivity operators. The relation to be as strong as is defined as follows:

- Given $\tau_{\Sigma}, \tau_{\Sigma}^{\prime} \in T_{\Sigma}$, operator $\tau_{\Sigma}$ is stronger than $\tau_{\Sigma}^{\prime}$ if for all $a, b \in I \subseteq \mathbb{R}$,

$$
\tau_{\Sigma}(a, b) \geq \tau_{\Sigma}^{\prime}(a, b) \text { where } \tau_{\Sigma} \neq \tau_{\Sigma}^{\prime}
$$

This relation is denoted as $\tau_{\Sigma} \sqsupseteq \tau_{\Sigma}^{\prime}$. Oppositely, it is said that operator $\tau_{\Sigma}^{\prime}$ is weaker than $\tau_{\Sigma}$.

- Given $\tau_{\Delta}, \tau_{\Delta}^{\prime} \in T_{\Delta}, \tau_{\Delta}$ is stronger than $\tau_{\Delta}^{\prime}$ if for all $a, b \in I \subseteq \mathbb{R}$,

$$
\tau_{\Delta}(a, b) \leq \tau_{\Delta}^{\prime}(a, b) \text { with } \tau_{\Delta} \neq \tau_{\Delta}^{\prime}
$$

This relation is denoted as $\tau_{\Delta} \sqsupseteq \tau_{\Delta}^{\prime}$. Oppositely, it is said that operator $\tau_{\Delta}^{\prime}$ is weaker than $\tau_{\Delta}$.

Therefore, a weak transitivity is less informative than a strong one. The objective is to keep the strongest transitivity possible when aggregating. In the following section we will collect the results of the studies made so far in order to keep transitivity in aggregation operators.

Before that, we introduce briefly a little proposition about transitive operators that we will use further.

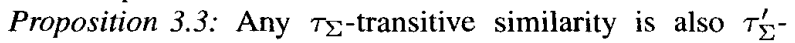
transitive, where $\tau_{\Sigma}^{\prime} \sqsubseteq \tau_{\Sigma}$. Any $\tau_{\Delta}$-transitive dissimilarity is also $\tau_{\Delta}^{\prime}$-transitive, where $\tau_{\Delta}^{\prime} \sqsubseteq \tau_{\Delta}$.

Proof: Using that if $\tau_{\Sigma}^{\prime} \sqsubseteq \tau_{\Sigma}$ then, for all $a, b$,

$$
\tau_{\Sigma}^{\prime}(a, b) \leq \tau_{\Sigma}(a, b)
$$

Trivially, for any $x, y, z \in X$,the following holds:

$$
s(x, y) \geq \tau_{\Sigma}\left(s(x, z), s(z, y) \geq \tau_{\Sigma}^{\prime}(s(x, z), s(z, y))\right.
$$

The proof is analogous for dissimilarities. 


\section{TRANSITIVITY IN AGgREgation}

Consider a set of $n \tau_{\Sigma}$-transitive similarities $s_{i}$, where $x_{i}, y_{i}, z_{i} \in X_{i}$ and $i \in\{1, \ldots, n\}$.

$$
s_{i}\left(x_{i}, y_{i}\right) \geq \tau_{\Sigma}\left(s_{i}\left(x_{i}, z_{i}\right), s_{i}\left(z_{i}, y_{i}\right)\right)
$$

And let $\Theta$ be an operator that aggregates the similarities. Thus,

$$
\Theta\left[s_{i}\left(x_{i}, y_{i}\right)\right]_{i} \geq \Theta\left[\tau_{\Sigma}\left(s_{i}\left(x_{i}, z_{i}\right), s_{i}\left(z_{i}, y_{i}\right)\right)\right]_{i}
$$

However we cannot assure that, for all $x, y, z$ in $X$, the following is fulfilled:

$$
\Theta\left[s_{i}\left(x_{i}, y_{i}\right)\right] \geq \tau_{\Sigma}\left(\Theta\left[s_{i}\left(x_{i}, z_{i}\right)\right]_{i}, \Theta\left[s_{i}\left(z_{i}, y_{i}\right)\right]_{i}\right)
$$

In [18], the concept of domination is introduced. We restrict here the definition for the specific case of dominating transitivity operators.

Definition 4.1 (domination): Consider an aggregation operator $\Theta$ and a transitive operator $\tau$. We say that $\Theta$ dominates $\tau$ if the following property holds:

- If $\tau$ is a similarity transitivity operator, for all $a_{i}, b_{i} \in I$ with $i \in\{1, \ldots, n\}$ :

$$
\Theta\left[\tau\left(a_{i}, b_{i}\right)\right]_{i} \geq \tau\left(\Theta\left[a_{i}\right]_{i}, \Theta\left[b_{i}\right]_{i}\right)
$$

- If $\tau$ is a dissimilarity transitivity operator, for all $a_{i}, b_{i} \in$ $I$ with $i \in\{1, \ldots, n\}$ :

$$
\Theta\left[\tau\left(a_{i}, b_{i}\right)\right]_{i} \leq \tau\left(\Theta\left[a_{i}\right]_{i}, \Theta\left[b_{i}\right]_{i}\right)
$$

Therefore, using this definition, the following Theorem is introduced:

Theorem 4.2 ([18, Theorem 9]): An aggregation operator $\Theta$ preserves $\tau$-transitivity if and only if $\Theta$ dominates $\tau$.

The question is: given a set of $\tau$-transitive similarities or dissimilarities, which aggregation operators do keep the transitivity? Another question: given a set of different $\tau_{i^{-}}$ transitivity similarities or dissimilarities, which will be the final aggregated transitivity? Let us answer the second question first.

Corollary 4.3: Given a set of $n$ similarity or $n$ dissimilarity functions, each one $\tau_{i}$-transitive $(i \in\{1, \ldots, n\})$, denote $\tau_{m}$ the weakest transitivity operator (see Expression 3.2). For any aggregation operator $\Theta$, the following is true:

- $\Theta$ dominates $\tau_{m} \Longleftrightarrow \Theta$ is $\tau_{m}$-transitive.

- $\forall i \in\{1, \ldots, n\} \quad \Theta$ dominates $\tau_{i} \Longrightarrow \Theta$ is $\tau_{m}$-transitive.

- $\Theta$ not dominates $\tau_{m} \Longleftrightarrow \Theta$ is not $\tau_{i}$-transitive, $\forall i \in$ $\{1, \ldots, n\}$.

Proof: The first and the third are direct consequences of Theorem 4.2. To prove the second one, use Proposition 3.3.

This result is very pessimistic because we cannot guarantee a stronger transitivity than the weakest of any similarity or dissimilarity aggregated. However, for the first question there are more interesting results for certain choices of $\tau$ and $\Theta$ (See [9], [5]), although not a general formula. Despite this, a general result for continuous transitivity operators can be collected in the following two theorems. Before, let us recall the definition of an Archimedean norm from Fuzzy Theory, because it is useful in this context.

A continuous and not idempotent norm is called Archimedean in fuzzy theory [19]. Following this nomenclature with transitive operators, we state that any Archimedean transitive operator can be defined using a function (called generator).

Definition 4.4 (Archimedean transitive operator): Let $\tau$ be a transitive operator defined on an interval $I, \tau$ is Archimedean only if there exists a function $f$ such that:

$$
\tau(a, b)=f^{-1}(f(a)+f(b))
$$

- If $\tau$ is a similarity transitive operator, then $f$ has to be a mapping from $I$ to $\mathbb{R}$, strictly decreasing, such that $f\left(s_{\max }\right)=s_{\max }$.

- If $\tau$ is a dissimilarity transitive operator, then $f$ has to be a mapping from $I$ to $\mathbb{R}$, strictly increasing, such that $f\left(\delta_{\min }\right)=\delta_{\min }$.

The only continuous and not Archimedean transitivity operators are min and max. They are a particular case that is covered with the following theorem:

Theorem 4.5: The only aggregation operator that dominates the transitivity operator min (and also it is dominated by) is min. The only aggregation operator that dominates the transitivity operator $\max$ (and also it is dominated by ) is $\max$.

The following result can be applied to any Archimedean transitive operator.

Theorem 4.6 ([18, Theorem 14]): Given an Archimedean transitive operator $\tau$ with a generator $f$. An aggregation operator $\Theta$ dominates $\tau$ if and only if

$$
f\left(\Theta\left[f^{-1}\left(a_{i}+b_{i}\right)\right]_{i}\right) \leq f\left(\Theta\left(f^{-1}\left(a_{i}\right)\right]_{i}\right)+f\left(\Theta\left[f^{-1}\left(b_{i}\right)\right]_{i}\right)
$$

This theorem is a generalization of that in [10, Theorem 3.3]. With this theorem we can test if our aggregation operator preserves a certain transitivity.

For example, if $f(z)=z$ then $\tau_{\Delta}(a, b)=a+b$. Using Theorem 4.6 any subadditive aggregation operator preserves transitivity.

$$
\Theta\left[a_{i}+b_{i}\right]_{i} \leq \Theta\left[a_{i}\right]_{i}+\Theta\left[b_{i}\right]_{i}
$$

For certain families of aggregation operators, some results can be inferred. Let us introduce the following family of aggregation operators:

$$
\Theta\left[z_{i}\right]_{i}=g\left(\sum_{i}^{n} w_{i} \cdot g^{-1}\left(\tilde{z}_{i}\right)\right)
$$

where $g$ is a monotonic function and $\sum_{i}^{n} w_{i}=1$.

Next we introduce a pair of theorems that can be useful used in combination of this family. The following theorem is a generalization of the one in [9], extended for any aggregation operator: 
Theorem 4.7: Given some aggregation operator $\Theta$ and a transitivity operator $\tau$, for a strictly increasing function $g$ :

$$
\begin{aligned}
\Theta^{\prime}(\vec{z}) & =g\left(\Theta\left[g^{-1}\left(z_{i}\right)\right]_{i}\right) \\
\tau^{\prime}(a, b) & =g\left(\tau\left(g^{-1}(a), g^{-1}(b)\right)\right)
\end{aligned}
$$

the following holds:

$$
\Theta \text { dominates } \tau \Longleftrightarrow \Theta^{\prime} \text { dominates } \tau^{\prime}
$$

Proof: Given some aggregation operator $\Theta$ and a similarity transitivity operator $\tau_{\Sigma}$, suppose that $\Theta$ dominates $\tau_{\Sigma}$. This means that $\forall a, b \in \operatorname{Dom}\left(\tau_{\Sigma}\right)$, the following is true:

$$
\Theta\left[\tau_{\Sigma}\left(a_{i}, b_{i}\right)\right]_{i} \geq \tau_{\Sigma}\left(\Theta\left[a_{i}\right]_{i}, \Theta[b]_{i}\right)
$$

Substitute $a_{i}^{\prime}=g\left(a_{i}\right)$ and $b_{i}^{\prime}=g\left(b_{i}\right)$

$$
\begin{gathered}
\Theta\left[\tau_{\Sigma}\left(g^{-1}\left(a_{i}^{\prime}\right), g^{-1}\left(b_{i}^{\prime}\right)\right)\right]_{i} \\
\tau_{\Sigma}\left(\Theta\left[g^{-1}\left(a_{i}^{\prime}\right)\right]_{i}, \Theta\left[g^{-1}\left(b_{i}^{\prime}\right)\right]_{i}\right)
\end{gathered}
$$

Applying $g^{-1} \circ g$ in some strategic places

$$
\begin{array}{r}
\Theta\left[g^{-1} \circ g \circ \tau_{\Sigma}\left(g^{-1}\left(a_{i}^{\prime}\right), g^{-1}\left(b_{i}^{\prime}\right)\right)\right]_{i} \\
\tau_{\Sigma}\left(g^{-1} \circ g \circ \Theta\left[g^{-1}\left(a_{i}^{\prime}\right)\right]_{i}, g^{-1} \circ g \circ \Theta\left[g^{-1}\left(b_{i}^{\prime}\right)\right]_{i}\right)
\end{array}
$$

Applying to both sides $g$,

$$
\begin{array}{r}
g \circ \Theta\left[g^{-1} \circ g \circ \tau_{\Sigma}\left(g^{-1}\left(a_{i}^{\prime}\right), g^{-1}\left(b_{i}^{\prime}\right)\right)\right]_{i} \\
g \circ \tau_{\Sigma}\left(g^{-1} \circ g \circ \Theta\left[g^{-1}\left(a_{i}^{\prime}\right)\right]_{i}, g^{-1} \circ g \circ \Theta\left[g^{-1}\left(b_{i}^{\prime}\right)\right]_{i}\right)
\end{array}
$$

Therefore, defining

$$
\begin{aligned}
\Theta^{\prime}(\vec{z}) & =g\left(\Theta\left[g^{-1}\left(z_{i}\right)\right]_{i}\right) \\
\tau_{\Sigma}^{\prime}\left(a^{\prime}, b^{\prime}\right) & =g\left(\tau_{\Sigma}\left(g^{-1}\left(a^{\prime}\right), g^{-1}\left(b^{\prime}\right)\right)\right)
\end{aligned}
$$

We get that $\Theta^{\prime}$ dominates $\tau_{\Sigma}^{\prime}$.

If we initially suppose that $\Theta$ does not dominate $\tau$, making the same substitutions the result is the $\Theta^{\prime}$ does not dominates $\tau_{\Sigma}^{\prime}$

The result in 9 is possible using [19, Theorem 3.13]. This is analogous for dissimilarities.

Theorem 4.8: Let $\Theta$ be an aggregation operator and $\tau$ a transitivity operator. Given that $\Theta$ dominates $\tau$, for any transitivity operator $\tau^{\prime}$ such that $\tau^{\prime} \sqsubseteq \tau$, it is true that $\Theta$ dominates $\tau^{\prime}$.

Proof: Using Theorem 4.2, if $\Theta$ dominates $\tau$ then the aggregation is $\tau$-transitive. If $\tau^{\prime} \sqsubseteq \tau$, then any similarity or dissimilarity $\tau$-transitive is also $\tau^{\prime}$-transitive using Proposition 3.3. Using again Theorem 4.2 we get that necessarily $\Theta$ also dominates $\tau^{\prime}$.

The foregoing theorems let us to choose some functions $g$ in Expression 6, such that a certain transitivity is kept. Thus, for instance, we can define the conditions for $g$ in order to keep the metric transitivity.

In the following section we introduce some examples of aggregation operators that dominates transitivity operators using the previous theorems.

\section{EXAMPLES}

Example 5.1: Let $\Theta$ be an aggregation operator

$$
\Theta\left[z_{i}\right]_{i}=\sum_{i}^{n} w_{i} z_{i}
$$

and $\tau_{\Delta}$ a dissimilarity transitivity operator.

$$
\tau_{\Delta}(a, b)=a+b
$$

It is easy to see that $\Theta$ dominates $\tau_{\Delta}$ because

$$
\sum_{i}^{n} w_{i}\left(a_{i}+b_{i}\right)=\sum_{i}^{n} w_{i} a_{i}+\sum_{i}^{n} w_{i} b_{i}
$$

Note that it is an strictly equality relation, not lower or equal relation. This implies that also $\tau_{\Delta}$ dominates $\Theta$. Using Theorem 4.7 and Expression 10, some domination relations can be extracted. In Table I some results are shown obtained with a few choices of function $g$ for the aggregation operators family described on Expression 5 .

Also, it is easy to see that $\Theta$ dominates $\tau_{\Sigma}$ when

$$
\tau_{\Sigma}(a, b)=a+b-s_{\max }
$$

Proceeding in the same way that with $\tau_{\Delta}$ we can get new dominance relations.

The previous example uses the addition as a dissimilarity transitivity operator. Dissimilarities that fulfill this transitivity (also called triangle inequality) are commonly known as metrics. The following proposition introduces a criterium to know if some aggregation operator keeps the triangle inequality.

Proposition 5.1: Let $\Theta$ be an aggregation operator of the family defined in Expression 5. If $g: I \longrightarrow I^{\prime}$ is subadditive then $\Theta$ preserves the transitive equality.

$$
\text { Proof: Given } \Theta\left[z_{i}\right]_{i}=\sum_{i}^{n} w_{i} z_{i} \text {, and } \tau_{\Delta}(a, b)=a+b \text {, }
$$

we know that $\Theta$ dominates $\tau_{\Delta}$. Using Theorem 4.7 we know that any modified aggregation operator given by Expression 6 dominates any modified transitive operator given by Expression 7. Using Theorem 4.8 we know that if the modified transitivity operator is stronger that $\tau_{\Delta}$, then the modified aggregation operator dominates $\tau_{\Delta}$. Thus,

$$
\begin{aligned}
g\left(g^{-1}(a)+g^{-1}(b)\right) & \leq a+b \\
g^{-1}(a)+g^{-1}(b) & \leq g^{-1}(a+b) \\
g(a)+g(b) & \geq g(a+b)
\end{aligned}
$$

Proved, $g$ is subadditive and $g^{-1}$ is superadditive.

Example 5.2: Given a set of $n$ metric dissimilarities $\delta_{i}$, (with $n \in \mathbb{N}$ and $i \in\{1, \ldots, n\}$ ), where $I \subseteq[1,+\infty$ ), and an aggregation operator $\Theta$ given by

$$
\Theta\left[z_{i}\right]_{i}=\ln \left(\sum_{i}^{n} w_{i} \cdot e^{z_{i}}\right) \quad \text { with } \sum_{i}^{n} w_{i}=1
$$

This aggregation keeps metric transitivity. It is easy to see that this is an aggregation operator that belongs to the family represented in Expression 5 with $g(z)=\ln (z)$. 
TABLE I

AGGREGATION OPERATORS THAT DOMINATE TRANSITIVE OPERATORS

\begin{tabular}{|c|c|c|}
\hline$g^{-1}(z)$ & $\Theta$ & $\tau(a, b)$ \\
\hline$z$ & $\sum_{i=1}^{n} w_{i} z_{i}$ & $a+b$ \\
$z^{2}$ & $\sqrt{\sum_{i=1}^{n} w_{i} z_{i}^{2}}$ & $\sqrt{a^{2}+b^{2}}$ \\
$\log z$ & $\prod_{i=1}^{n} z_{i}^{w_{i}}$ & $a \cdot b$ \\
$z^{-1}$ & $\frac{a b}{a+b}$ \\
$z^{\alpha}$ & $\left(\sum_{i=1}^{n} w_{i}^{n} w_{i} z_{i}^{\alpha}\right)^{1 / \alpha}$ & $\sqrt[\alpha]{z^{\alpha}+b^{\alpha}}$ \\
\hline
\end{tabular}

\section{CONCluSions}

There are several definitions of similarity, dissimilarity or metric, and often these concepts are misused, or mixed. In this sense, something similar happens with aggregation and transitivity. This is a wide field of study that unifies many concepts (e.g. from metrics to fuzzy relations) but has been usually ignored and where there is not much research. Results in this paper are a general case of the studies made on aggregation operators used to keep metric transitivity. This is, the similarity and dissimilarity framework has metric distances (metric dissimilarities) as a particular case of dissimilarities. We think that research in this field can lead to similar results with non-metric dissimilarities or similarities.

In this paper we tried to clarify some concepts and to link similarity and dissimilarity functions and their properties with the effects of aggregation on transitivity. We also have collected the most recent results in transitive aggregation. Finally, we made a little contribution with a generalization of a theorem.

\section{ACKNOWLEDGMENT}

This document has been granted by the Interministerial Council of Science and Technology (CICYT) of Spain, within the project DPI2002-03225 and by the Spanish Ministry of Education, Culture and Sport (MECD) with the grant FPU AP2001-3735.

\section{REFERENCES}

[1] J. Gower, "A general coefficient of similarity and some of its properties," Biometrics, no. 27, 1971.

[2] F. Klawonn and J. L. Castro, "Similarity in fuzzy reasoning," Mathware \& Soft Computing, vol. 2, pp. 197-228, 1995.

[3] A. Tversky, Features Of Similarity. Psycological Review, July 1977, vol. 84(4), pp. 327-352.

[4] S. Santini and R. Jain, "Similarity measures," in IEEE Transactions On Pattem Analysis and Machine Intelligence, 1999.

[5] P. Fonck, J. Fodor, and M. Roubens, "An application of aggregation procedures to the definition of measures of similarities between fuzzy sets," Fuzzy Sets and Systems, vol. 97, pp. 67-74, 1998.

[6] D. Bridge, "Defining and combining symmetric and asymmetric similarity measures," in Advances in Case-Based Reasoning (Procs. of the Fourth European Workshop on Case-Based Reasoning), B.Smyth and P.Cunningham, Eds. Springer, 1998, pp. 52-63.

[7] A. Ferguson and D. Bridge, "Generalised weighting:a generic combining form for similarity metrics," in Procs. of the Eleventh Irish Conference on Artificial Intelligence \& Cognitive Science (AICS'2000), J. Griffith and C. O'Riordan, Eds., 2000, pp. 169-179.

[8] R. Mesiar and M. Komomíková, "Aggregation operators," in Proceeding of the XI Conference on applied Mathematics PRIM' 96, D. Herceg and K. e. Surla, Eds. Novi Sad: Institute of Mathematics, 1997, pp. 193211

[9] S. Ovchinnikov, "Aggregating transitive fuzzy binary relations," International Journal of Uncertainy, Fuгzyness Knowledge-based Systems, vol. 3, no. 1, pp. 47-55, 1992.

[10] J. Fernández Salido and S. Murakami, "On $\beta$-precision aggregation," Fuzzy Sets and Systems, vol. 139, 2003.

[11] L. A. Zadeh, "Similarity relations and fuzzy orderings," Inform. Sci., vol. 3, pp. 177-200, 1971

[12] H. Osborne and D. Bridge, "Similarity metrics: A formal unification of cardinal and non-cardinal similarity measures," in ICCBR, 1997, pp. 235-244.

[13] M. D. Cock and E. Kerre, "On (un)suitable relations to model approximate equality," Fuzzy Sets And Systems, vol. 133, pp. 137-153, 2003.

[14] F. Klawonn, "Should fuzzy equality and similarity satisfy transitivity?" Fuzzy Sets And Systems, vol. 133, pp. 175-180, 2003.

[15] A. Tversky and I. Gati, Similarity, Separability and the Triangle Inequality. Psycological Review, July 1982, vol. 86, pp. 123-154.

[16] J. L. Marichal, "Aggregation operations for multicriteria decision aid." Ph.D. dissertation, University of Liège, Belgium, 1998.

[17] B. Schweizer and A. Sklar, Probabilistic Metric Spaces. Amsterdam: North-Holland, 1983.

[18] S. Saminger, R. Mesiar, and U. Bodenhofer, "Domination of aggregation operators and preservation of transitivity," Internat. J. of Uncertainity in Fuzziness Knowledge-Based Systems, vol. 10, pp. 11-35, 2002.

[19] G. Klir and B. Yuan, Fuzzy Sets and Fuzzy Logic: Theory and Applications. Pearson Education POD, 1995, ch. 3. 